\title{
The Dedicated Aerosol Retrieval Experiment (DARE). Scientific requirements for a dedicated satellite instrument to measure atmospheric aerosols
}

\author{
Rob Decae ${ }^{\mathrm{a}}$, Grégory Bazalgette Courrèges-Lacoste ${ }^{\mathrm{b}}$, Gerrit de Leeuw ${ }^{* a}$ \\ ${ }^{a}$ TNO Physics and Electronics Laboratory, P.O. Box 96864, 2509 JG The Hague, The Netherlands; \\ TNO TPD, P.O. Box 155, Delft, The Netherland
}

\begin{abstract}
DARE (Dedicated Aerosol Retrieval Experiment) is a study to design an instrument for accurate remote sensing of aerosol properties from space. DARE combines useful properties of several existing instruments like TOMS, GOME, ATSR and POLDER. It has a large wavelength range, 330 to $1000 \mathrm{~nm}$, to discriminate between aerosol types. The wide swath will enable daily global coverage of the earth at a pixel size of $5 \times 5 \mathrm{~km}^{2}$ (nadir). The instrument will have three viewing angles, looking forward, at nadir and backward. These angles will facilitate the separation of atmospheric and surface contributions to the satellite signal. Multiple views will also help to determine the height and thickness of aerosol layers. Full polarization information will be measured for at least one of the viewing angles and at many of the available wavelengths. Polarization helps to separate surface/atmosphere signals and it contains valuable information on the shape of aerosol particles. Cloud detection will be enhanced by adding thermal infrared detectors at the same spatial resolution. Simulations and tests were performed to optimize the current design of the instrument. The expected performance of DARE in comparison to other instruments will be discussed.
\end{abstract}

Keywords: Aerosol, DARE, multiple view, polarization,

\section{INTRODUCTION}

The effect of aerosols is the largest uncertainty in climate ${ }^{1}$ : due to scattering of solar irradiation they cause cooling, of similar magnitude as warming due to greenhouse gases, and thus balancing the greenhouse effect. Other aerosol types absorb radiation, affecting the atmospheric circulation pattern and reinforcing the greenhouse effect. Aerosol-cloud interactions add an even larger uncertainty referred to as the indirect aerosol effect. Another consequence of the aerosol optical properties is that they influence airborne and space-based Earth observation with electro-optical (EO) sensors such as (multi-spectral) radiometers, leading to uncertainties in surface (water, land and, vegetation) observations. Atmospheric correction of EO satellite observations is inaccurate because of uncertainties in the aerosol effects. This is a recurrent problem because, for the retrieval of aerosol effects, the surface properties need to be accurately known, whereas the aerosols in turn influence the retrieval of surface properties. Other effects of aerosols are, for example, their influence on UV radiation at ground level, respiratory health effects of dust, transport of pollutants and the consequences for air quality, water quality and eco-systems, visibility reduction. In extreme cases (biomass burning and fires) airports must be closed for safety. Aerosols are also important for military applications because they affect the EO propagation environment including sensor performance for target detection and recognition.

The strong variability of aerosol physical and chemical properties makes it extremely difficult to produce reliable a priori predictions of their occurrence and effects, or even to use emission data bases for primary emissions and gas phase emission

\footnotetext{
*deleeuw@,fel.tno.nl; phone+31-70-3740462, fax +31-70-3740654;
} 
inventories to estimate effects on secondary aerosol formation. Models are under development, based on chemistry transport models that are using sophisticated chemical reaction schemes, analyzed meteorological fields and emission data bases (or inventories) as input. The results are promising, but many uncertainties remain, especially due to variability of primary aerosol emissions, and also due to the complexity of heterogeneous chemistry involving aerosols. Improvement could be obtained from data assimilation, using observed aerosol fields.

This requires reliable data with good spatial and temporal resolution. Ground based observations are labour intensive, and usually only available in very small parts of the world, aeroplane measurements are too expensive to provide good coverage in space and time.

A cost effective method would be the use of satellites. However, although methods are developed to retrieve aerosol properties from multispectral satellite measurements since more than two decades, until recently only over the oceans data were available on a routine basis and over land only an aerosol index, for absorbing aerosols, existed. TNO-FEL was the first to retrieve aerosol properties over land $^{2}$ and the scientific results ${ }^{3}$ are currently used to convert the algorithm for application on a routine basis as a data product for daily use ${ }^{4}$.

\begin{tabular}{|c|c|c|c|c|c|c|c|c|}
\hline Instrument & Orbit & $\begin{array}{l}\text { Swath } \\
\text { (km) }\end{array}$ & $\begin{array}{l}\text { Spatial } \\
\left(\mathrm{km}^{2}\right)\end{array}$ & Spectral & Coverage & $\begin{array}{l}\text { Polarizati } \\
\text { on }\end{array}$ & $\begin{array}{l}\text { Viewing } \\
\text { angles }\end{array}$ & $\begin{array}{l}\text { Co- } \\
\text { location }\end{array}$ \\
\hline ATSR & Polar & 512 & $1 \times 1$ & $\begin{array}{l}\text { VIS, NIR, } \\
\text { TIR }\end{array}$ & 3 days & $\mathrm{N}$ & 2 & $\mathrm{Y}$ \\
\hline AVHRR & Polar & 2000 & $1 \times 1$ & $\begin{array}{l}\text { VIS, NIR, } \\
\text { TIR }\end{array}$ & 1 day & $\mathrm{N}$ & 1 & $\mathrm{Y}$ \\
\hline GOME & Polar & 960 & $340 \times 40$ & $240-790$ & 3 days & $\mathrm{N}$ & 1 & $\mathrm{Y}$ \\
\hline MERIS & Polar & 1150 & $0.3 \times 0.3$ & $400-1050$ & 3 days & $\mathrm{N}$ & 1 & $\mathrm{Y}$ \\
\hline MISR & Polar & 360 & $\begin{array}{l}0.275 \times 0.27 \\
5\end{array}$ & $\begin{array}{ll}3 & \text { VIS, } \\
1 \text { IR } & \end{array}$ & 7 days & $\mathrm{N}$ & 9 & $\bar{Y}$ \\
\hline MODIS & Polar & 2330 & $0.5 \times 0.5$ & $400-1400$ & 2 per day & $\mathrm{N}$ & 1 & $\mathrm{Y}$ \\
\hline MSG & $\begin{array}{l}\text { Geo- } \\
\text { stationary }\end{array}$ & Earth disk & $\begin{array}{l}3 \times 3 \\
\text { (equator) }\end{array}$ & $\begin{array}{l}\text { VIS, NIR, } \\
\text { TIR }\end{array}$ & $15 \mathrm{~min}$ & $\mathrm{~N}$ & 1 & $\mathrm{Y}$ \\
\hline OMI & Polar & 2600 & $13 \times 24$ & $270-500$ & 1 day & $\mathrm{N}$ & 1 & $\mathrm{Y}$ \\
\hline POLDER & Polar & 2200 & $6 \times 7$ & $\begin{array}{l}\text { VIS, } \\
\text { NIR } 7 \text { Ch }\end{array}$ & 1 day & $\mathrm{Y} \mathrm{(3} \mathrm{Ch)}$ & \pm 12 & $\mathrm{~N}$ \\
\hline $\begin{array}{l}\text { SCIAMAC } \\
\text { HY }\end{array}$ & Polar & 960 & $30 \times 60$ & $240-2380$ & 3 days & Y? & $1+$ & $\mathrm{Y}$ \\
\hline TOMS & Polar & 2800 & $42 \times 42$ & $\begin{array}{ll}6 & \text { UV } \\
\text { bands } & \end{array}$ & 1 day & $\mathrm{N}$ & 1 & $\mathrm{Y}$ \\
\hline DARE & Polar & 2600 & $5 \times 5$ & $\begin{array}{l}330-1000, \\
\text { TIR }\end{array}$ & 1 day & $\mathrm{Y}$ & 3 & $\mathrm{Y}$ \\
\hline
\end{tabular}

Table 1: Instruments used for aerosol retrieval

A number of other instruments than ATSR-2 is currently used for the retrieval of aerosol properties over land and over water, see table 1 . Yet the situation is not ideal because the instruments that are used today for aerosol retrieval are mostly designed 
with other primary scientific goals, for example, to measure sea surface temperature or atmospheric gas concentrations. A dedicated instrument aimed at aerosols would be able to reduce the large uncertainties connected to this kind of remote sensing.

In this contribution, we discuss the scientific requirements for a dedicated aerosol instrument. The technical specifications are discussed elsewhere. 5

\section{AEROSOL RETRIEVAL}

Satellite aerosol retrieval is a largely under-determined problem. Aerosols can be characterised by the shape of the particles, their size distribution and their refractive index. The vertical distribution of an aerosol layer can be described by a minimum and a maximum height and a distribution function (e.g., homogeneous or exponentially decreasing).

The land or water surface under the aerosol layer is another unknown factor. Surface reflectance depends on many factors and therefore is very hard to determine accurately. The reflectivity of the surface changes with the wavelength and angular configuration. The surface reflectance is often much higher than the aerosol signal. Choosing wavelengths with low surface reflectance (UV over land, NIR over water) can improve retrievals very much.

However, the instruments that are currently flying haven't been optimised to measure aerosols. The right combination of measurements could resolve many of the unknown factors or at least reduce some measurement errors.

\subsection{Parameters that can be measured by a satellite instrument.}

Traditional aerosol retrieval only uses the spectral reflectance to determine the optical thickness. There is a need of much $a$ priori information in order to make this inversion. Each independent piece of information that can be added to the measurements will reduce this a priori input. Instruments with multiple view capabilities can, in combination with the right wavelength range, measure the surface reflectance over land. The addition of only one more viewing angle like in the ATSR instruments, can already reduce surface-induced errors dramatically. More views may also help in determining height and thickness of aerosol layers.

Polarisation is not commonly measured by satellite instruments. For aerosol retrieval however, polarisation information can be of great importance ${ }^{7}$. Theoretical studies have shown that polarisation could yield information on the shape of particles and probably also on the size and refractive index. Separation of surface and atmospheric signals might be much easier with additional polarised information available. Measurements from POLDER, one of the few instruments that measure polarisation at more than one wavelength has produced very useful aerosol and surface information. Detection of some types of clouds (e.g. high cirrus ice clouds) will be easier with polarised reflectances.

\section{INSTRUMENT REQUIREMENTS}

For the definition of the instrument requirements a choice has to be made as regards the application of the results. For DARE daily global coverage with accurate aerosol information contributing to studies on climate and long range transport has been the primary consideration. Considerations for the DARE design are discussed below.

\subsection{Orbit}

The choice for an orbit is strongly related to the type of intended measurements and the users of the data. Global monitoring requires global coverage and therefore eliminates geo-stationary orbits. If regional sources, sinks and transport data are needed, such an geo-stationary satellite would be the more obvious choice.

A geo-stationary orbit has a number of disadvantages as compared to a polar orbit:

- multiple viewing angles are not possible 
- Polarisation information can only be gathered for one fixed viewing direction per pixel; although the change in solar angle will provide interesting changes, the temporal variability of the aerosol properties and in particular the aerosol layering diurnal changes and associated concentration changes and humidity effects prohibit the reliable use of this feature.

- The height of the satellite orbit combined with the requirement for a small ground pixel will result in a very expensive instrument.

\subsection{Swath width}

Swath width is mainly an issue for satellites in a polar orbit. For daily global coverage, the swath should be wide enough to cover the equator in 14 orbits. The number of orbits is connected to the height of the orbit. The circumference of the earth at the equator is about $40000 \mathrm{~km}$ so this would result in a swath of about $2800 \mathrm{~km}$. The swath of many instruments with daily global coverage is usually smaller; full daily coverage is not obtained near the equator. A very wide swath results in large pixels or poor signal to noise ratio near the edges of the swath.

\subsection{Spectral range and resolution}

The spectral range that is useful for aerosol retrieval ranges from the UV to the near IR. Unlike for gas retrievals, the spectral resolution needed for aerosol retrieval is not very high. The change of aerosol reflectance with the wavelength is gradual and therefore can be accurately described with a small number of separate wavelength bands over the whole range from UV to NIR. Ideally, this range would extend from about $330 \mathrm{~nm}$ to $2 \mu \mathrm{m}$. Strong absorption features of ozone, $\mathrm{NO}_{2}$, ring lines and other gasses should be avoided and therefore a resolution between 2 and $5 \mathrm{~nm}$ would be desirable.

The need for polarization information implies the use of a prism instead of a grating system for wavelength separation. The use of a prism results in a decrease in spectral resolution with increasing wavelength. The size (number of wavelength pixels) of the detector and the needed signal to noise ratio determine the size of the wavelength bands. With a prism, the choice of 2 $\mathrm{nm}$ wide bands in the UV will result in $40 \mathrm{~nm}$ wide bands in the NIR. With this width these bands will contain a number of gas absorption lines. In some cases, the signal will have to be corrected for gas absorption, e.g. ozone.

If polarization information is needed for each ground pixel and each wavelength, each polarization direction requires the same size detector. Detectors represent a significant part of the costs of an instrument. The use of standard silicon detectors will reduce costs but will limit the upper wavelength to about $1000 \mathrm{~nm}$. Larger detectors of this type have $2000 \mathrm{x} 2000$ pixels. This would be enough to accommodate two polarisation directions on one CCD at full spatial and spectral resolution.

\subsection{Multiple viewing angles}

The usefulness of multiple viewing angles for aerosol retrieval has been demonstrated for ATSR-2, Polder and MISR. More views of the same scene in a short time period provides the possibility to determine the surface reflectance thus reducing the uncertainty in the retrievals dramatically.

In addition to this it might be possible to derive some information from the different views on the vertical structure of the aerosol layers. Errors in height and thickness of the layers can be an important source of errors in the UV wavelength range ${ }^{8}$.

\subsubsection{The number of cameras needed in DARE.}

The number of viewing directions and the exact angle of the different cameras depends on several factors. The phase function of aerosols depends on the refractive index and thus on the aerosol composition. Observation of characteristic peaks will be optimal if the viewing angle is close to the top of a peak. Obviously, because the satellite is moving, the viewing angle should be adjusted continuously to remain at this peak level. It is therefore better to choose a few viewing-angles that are useful to measure the main aerosol types at their usual positions on earth. The most problematic type of aerosol to retrieve is mineral dust. It is non-spherical, partly absorbing depending on its origin, and it can be transported in high and dense layers over large distances. Optimization of the viewing angles for dust in the areas where it usually occurs should lead 
to large improvements in the retrieval of this type of aerosol. This choice has to be made in connection to the choice for a certain type of orbit.

Results will get better when more viewing angles are available, but the number of cameras is obviously limited by financial restrictions. For the elimination of the surface, two viewing angles may provide information. ${ }^{5}$ To measure the phase function properly, especially for non-spherical particles, a minimum of three angles is highly desirable: one at nadir and the others pointing forward and backward in flight direction.

\subsubsection{Values for the off-nadir camera angles}

The choice for a 3-camera system has implications for the choice of camera angles. Polarisation measurements are most interesting in sideward scattering angles between 60-120 degrees. For intensity (unpolarised) measurements it is important to look at different scattering angles, especially measurements close to 180 degrees are desirable to determine the shape of the phase function.

A large part $(>50 \%)$ of the measured light is single scattered with one particular scattering angle that is dependent on the viewing geometry of the sensor and the position of the sun. This will be called the single scattering angle $\theta$. Measured intensity and polarisation are both dominated by F11 and F12 at $\theta$. Inversely, a measurement at $\theta$ contains information about F11 and F12 mainly at $\theta$.

The single scattering angle was calculated for different viewing geometries en solar positions. From this, it was tried to derive suitable scattering angle combinations at interesting latitudes.

The strongest sources for mineral dust aerosol are found at $+15^{\circ}$ to $+30^{\circ}$ latitude (Mexico/Sahara) and further north towards Asia $+40^{\circ}$ to $+50^{\circ}$ (Gobi). Industrial aerosols and biomass burning aerosols are concentrated on the Northern Hemisphere as well, so it is advantageous to optimise for it.

The backward viewing direction: 53 degrees is a good choice especially for polarisation measurements. In this viewing direction scattering angles from $60^{\circ}$ to $120^{\circ}$ where polarisation contains most information about mineral dust, are reached at latitudes with an arid climate in autumn, winter and spring. In summer these scattering angles are reached a bit further north, which is fine since then the inter-tropical convergence zone shifts to the North and brings rain to the Sahel area.

The forward viewing angle may be chosen smaller. The situation is less clear - every choice has advantages and disadvantages. A larger angle is problematic. Then molecular scattering becomes strong and one can see less deep into the atmosphere. There is no particular advantage for a symmetric choice. A clear advantage of a smaller angle is that 3 different air masses are observed.

A scattering angle close to $180^{\circ}$ is desirable for the intensity measurements. In winter this requires a large forward viewing angle, while in summer the scattering angle maximum shifts to extreme high latitudes where aerosol loads are usually small. Therefore, there is a trade off between summer and winter. An angle of approximately $40^{\circ}$ would be a good compromise.

\subsubsection{Vertical profile information}

The occurrence of aerosol layers higher in the atmosphere can be a large error source especially for the shorter wavelengths. Observation of these layers from different angles could provide some clue of the height and thickness of the layer. Initial calculations show that the reflectance ratio depends on the height and the thickness of the layer.

\subsection{Polarisation}

The potential of using polarisation information in aerosol retrieval is large. With this information, a (partially) independent set of measurement parameters is added and this should lead to a smaller need for a priori input. The use of polarisation in earth remote sensing is relatively new. POLDER, a French instrument flying on the Japanese ADEOS platforms, provides 
polarisation information for three wavelengths and a large number of viewing angles. This combination of viewing angles and polarisation has proven to be a very powerful in some of the aspects of aerosol retrieval.

Possible uses for these parameters are:

- better separation of surface and atmospheric reflectances

- better cloud detection

- estimation of aerosol particle shapes

Because of the still limited use of polarisation, the knowledge of the remote sensing community on this subject is limited. Researchers at the AMOLF institute in Amsterdam are polarisation experts. They have been measuring polarisation of aerosols in great detail and they have specialised in characterisation of non-spherical particles. Errors caused by assuming the wrong shape for particles is potentially one of the biggest errors in aerosol retrieval ${ }^{9}$.

Polarisation issues in the design of DARE have been studied in cooperation with Veihelmann et al. ${ }^{7}$. The main aim of the first analysis was to determine the desired spectral and directional information extend of the polarisation data. The amount of needed data has a large impact on the required number of detectors in DARE.

The main questions in relation to the possibilities of polarisation were:

- What additional information can be expected from polarisation measurements?

- Is polarisation information needed for all available wavelengths?

- How many Stokes parameters are relevant for aerosol retrieval?

- Is polarisation information needed for all viewing angles?

The transformation of an optical signal by an aerosol is described by a $4 * 4$ Mueller matrix, see Figure 1 . The four Stokes parameters describe:

- I: the unpolarised reflectance

- Q: The linear polarised reflectance in the same plain

- U: They linear polarised reflectance in other plains

- V: circular polarised reflectance

Spherical polarisation does not occur in natural light; F4 components of the Mueller matrix are not needed and V can be neglected. Polarisation features are usually presented as a (negative) ratio between the polarised and non-polarised signal (e.g. -F12/F11) plotted against the scattering angle. Results are presented in Veihelmann et al. ${ }^{7}$. Application of the method lead to the conclusion that there is a need for spectral polarisation information over the whole wavelength range of DARE. Both I, Q and $\mathrm{U}$ are needed. This information is needed in all three viewing directions.

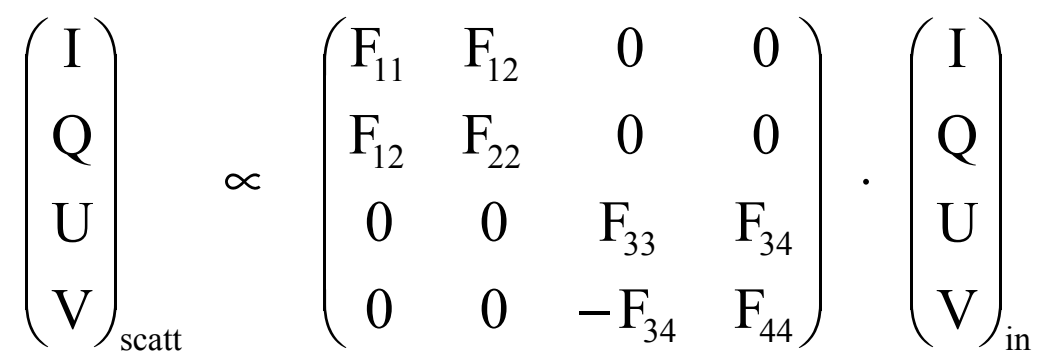

Figure 1: Transformation of the different Stokes parameters by the Mueller matrix.

\subsection{Signal to noise ratio}

The retrieval error depends on many factors like cloud contamination, errors in the estimation of the surface reflectance and the a priori information. The instrument itself will also introduce some error sources due to radiometric calibration. The signal to noise level is the first limitation in quality of the signal; it sets the minimum error level for the whole retrieval 
process. The desired signal to noise level $(\mathrm{S} / \mathrm{N})$ depends on the maximum allowed error of the product. For the AOD, an error of $5 \%$ or less would be very desirable.

The effects of changing S/N level on the final retrieval without any other sources of errors can be studied with a simple version of a retrieval algorithm. Random noise can be applied to simulated input data and results of the retrieval with and without noise can be compared.

For the evaluation of the the $\mathrm{S} / \mathrm{N}$ requirements for DARE, a test version of the OMI retrieval algorithm ${ }^{9}$ was constructed. This algorithm can add all kinds of errors and noise to the input signal. The output for a standard sample of 48 different angle combinations was used. The wavelength of DARE will probably be larger than that of OMI, which will lead to a better performance in the retrievals but the spectral sampling will be lower thus introducing a larger error due to gas absorption and ring effect. The results from the OMI simulator are therefore probably a good indication for the $\mathrm{S} / \mathrm{N}$ levels needed for DARE.

The OMI beta algorithm was used with the OMI IS2 aerosol type (Urban/Industrial type with medium sized particles and low absorption). ${ }^{9}$ The input or simulated measurement data were taken from the LUT for this type of aerosol. The signal to noise level was set to 200, 400, 800 and 1600 and the algorithm was run for two AOD (0.1 and 1.0) levels and the 48 test configurations. The resulting retrieved AOD for each of these 48 cases were compared to the retrieved AOD without the introduced noise.

The results of these exercises show that the errors for $\mathrm{AOD}=1.0$ are within the $5 \%$ interval at $\mathrm{S} / \mathrm{N}$ levels higher than 400 . At lower and more usual values of $\mathrm{AOD}=0.1$, the $\mathrm{S} / \mathrm{N}$ should be higher than 800 to reach the same relative results.

A signal to noise level of 800 will be difficult to reach especially for non-nadir viewing cameras. As the $\mathrm{S} / \mathrm{N}$ fixes the minimum error level, the final retrieval error will probably be much higher. The 48 cases contain extreme values for all angles; retrieval for these extreme angles has higher errors. This implies that mean retrieval error for a scene will be less than half of the relative error range.

The signal in the UV will be lower than in at longer wavelengths but the spectral sampling is higher. Binning of a few wavelength bands in the UV might increase the $\mathrm{S} / \mathrm{N}$ to the desired level.

\subsection{Cloud detection}

Cloud detection is a major issue in aerosol retrieval. It is a challenge to find the right balance between rejection of cloudcontaminated pixels without the loss of aerosol information. Certain types of clouds have a spectral behaviour close to that of some aerosol types. Other cloud types like thin cirrus are difficult to detect but may have a large impact on retrieval quality. The availability of infrared channels is very important for the right discrimination between aerosols and clouds. Polarisation is potentially useful in detecting ice clouds such as high cirrus. An overview of cloud detection techniques that are used in some of the instruments mentioned in Table 1 is presented by Decae ${ }^{8}$.

This overview shows that cloud detection and discrimination between clouds and aerosols can be done in a number of ways. The main tools are differences in visible and thermal infrared channels (MODIS), polarisation (POLDER) and multipleviewing angles (MISR). None of these methods is perfect for all circumstances but the combination of the different techniques should result in excellent cloud masking.

This would require some TIR channels in addition to the aerosol observation system. These TIR detectors are probably only necessary for one viewing direction. Preferred wavelengths would be 11 and $12 \mu \mathrm{m}$. These wavelengths are also used in the Apollo scheme for the ATSR cloud detection. Polarisation in a few channels will enable the same testing that POLDER uses while more viewing angles might facilitate the use of a MISR-like detection scheme. 


\section{CONCLUSIONS}

A study of the existing instruments with aerosol measuring capability provided a list of useful features for such a DARE instrument. The analysis of these features in section 3 has lead to a number of requirements and restrictions for the design of a dedicated aerosol retrieval instrument. The choice for the type of orbit determined many of the other properties of the instrument. It was decided that DARE will be a monitoring instrument with (daily) global coverage over non-polar areas. The inclination of the orbit can probably be chosen in such a way that e.g. Europe is covered more than once a day. Daily global coverage is only possible with a swath width that is large enough e.g. $2600 \mathrm{~km}$.

These criteria and the considerations and calculations presented in Chapter 3 have lead to the instrument requirements for a dedicated aerosol spectrometer. defined as:

- A spectral range from $330-1000 \mathrm{~nm}$ with a spectral resolution from $2 \mathrm{~nm}$ (UV) to $>30 \mathrm{~nm}$ (NIR)

- Observation in at least 3 polarization directions (Stokes parameters)

- A FOV in swath direction of $>114^{\circ}$.

- The wide FOV will enable daily global coverage of the earth at a pixel size of $5 \times 5 \mathrm{~km}^{2}$ (Nadir). From a satellite altitude of $800 \mathrm{~km}$ (polar orbit).

- Observation in at least 3 viewing directions (backwards $\left(-53^{\circ}\right)$, nadir $\left(0^{\circ}\right)$, forward $\left(+53^{\circ}\right)$ ).

Each viewing channel is a module consisting of a prism imaging spectrometer with 2 detector arrays which are used to measure a spectrum for each of the 3 polarization directions.

As a modular system, implementation of changes in the number of observation directions can be easily realized.

The three viewing angles will allow for the separation of atmospheric and surface contributions to the satellite signal. Multiple views will also help to determine the height and thickness of aerosol layers.

Full polarization information will be measured for at least one of the viewing angles and all available wavelengths. Polarization sensitivity not only helps to separate surface/atmosphere signals but it also contains valuable information on the shape properties of the aerosol particles.

The resulting properties of DARE have been included in Table 1. Comparison of the specifications of DARE with those of other instruments shows the improvement and expected performance of this instrument. These requirements have been translated into a DARE optical concept presented in the accompanying paper by Smorenburg et al. ${ }^{5}$

\section{ACKNOWLEDGEMENTS}

The authors thank Ben Veihelmann, Hester Volten and Wim van der Zande from AMOLF (Amsterdam, The Netherlands) for their contributions to the polarization studies. DARE is an internal TNO project.

\section{References}

1. IPCC (Intergovernmental Panel on Climate Change), Climate change 2001: The scientific basis. Houghton J. T., Y. Ding, D. J. Griggs, M. Noguer, P. J. van der Linden, X. Dai, K. Maskell, and C. A. Johnson (eds.). Cambridge University Press, Cambridge, United Kingdom and New York, NY, USA, 2001.

2. J.P. Veefkind, G. de Leeuw and P.A. Durkee (1998). Retrieval of aerosol optical depth over land using two-angle view satellite radiometry during TARFOX. Geophys. Res. Letters. 25(16), 3135-3138. 
3. C. Robles Gonzalez, Retrieval of Aerosol Properties using ATSR-2 Observations and their Interpretation, PhD thesis, Univ. of Utrecht, June 2003.

4. See: www.temis.nl

5. K. Smorenburg, G. Bazalgette Courreges-Lacoste, R. Decae, A. Court, G. de Leeuw and H. Visser, DARE: Dedicated aerosol retrieval instrument, SPIE-proceedings 5235 (this volume), Barcelona, Sept. 2003.

6. A. Court, K. Smorenburg, G. Bazalgette Courreges-Lacoste, H. Visser, G. de Leeuw and R.J. Decae, DARE: a dedicated aerosol retrieval instrument, Proceedings of the 54th International Astronautical Congress, Bremen, Germany, September 29 - October 3, 2003

7. B. Veihelmann, H. Volten and W.J. van der Zande, Light Reflected by an Atmosphere Containing Irregular Mineral Dust Aerosol, submitted for publication, 2003.

8. R. Decae, Dedicated Aerosol retrieval Experiment (DARE): Instrument requirements, TNO Physics and Electronics Laboratory, Report, in preparation, 2003.

9. O. Torres, R. Decae, P. Veefkind and G. de Leeuw, OMI Aerosol Retrieval Algorithm. In: P. Stammes and R. Noordhoek (eds.) OMI Algorithm Theoretical Basis Document Volume III: Clouds, Aerosols and Surface UV Irradiance, ATBD-OMI03, pp. 46-71, 2002. 